\title{
Modulation of the Spontaneous and Evoked Discharges of Ventral Posterior Thalamic Neurons During Shifts in Arousal
}

\author{
THOMAS J. MORROW* $\ddagger^{*}$ AND KENNETH L. CASEY*†† \\ Departments of Physiology* and Neurology, $\dagger$ and the Neurology Research Laboratories $\ddagger$ \\ University of Michigan and V. A. Medical Center. Ann Arbor. MI 48109
}

\begin{abstract}
MORROW, T. J. AND K. L. CASEY. Modulation of the spontaneons and orohed discharges of ventral postertion thatamic ncurons during shifts in arousal. BRAIN RES BULL 21(3) 433-438, 1988. - The responses of 154 ventral posterior thalamic neurons to a variety of somatic stimuli and to electrical stimulation of the midbrain spinal lemniscus were recorded in the awake squirrel monkey during varying states of arousal. Many VP (42/93) neurons showed changes in somatosensory responsiveness which correlated with shifts in arousal. Arousal related modulation (ARM) of somatic responses were not selective for any specific stimulus modality. Most cells $(\mathrm{N}=36)$ responded maximally during quiet waking with responses significantly reduced during drowsiness or periods of waking movement. Other neurons $(N=5)$ responded maximally during drowsiness, and gave decreased responses as the level of arousal increased. Similar changes were seen for neurons driven by spinal lemniscal (SL) stimulation. All changes in evoked responses were independent of prestimulus background discharge frequency. At least one site of ARM takes place at the level of the VP thalamus.
\end{abstract}

Thalamus Arousal Somatosensory modulation Single unit recording Behavioral neurophysiology

Awake monkey

OUR sensory systems are continually bombarded by inputs from which we select information as a basis for action. Perception of a sensory stimulus can be altered during shifts of attention, vigilance or arousal. It is therefore reasonable to hypothesize that certain properties of the response of a neuron to a specific sensory stimulus should covary with changes in an animal's behavioral state. Accordingly, the somatosensory responses of ventral posterior (VP) thalamic neurons would be expected to vary with the level of arousal in unanesthetized, behaving animals.

With few exceptions, most studies of the somatosensory properties of thalamic neurons have been done in anesthetized animals. This work has suggested that most neurons in the ventral posterior part of the thalamus possess what have come to be designated as "lemniscal properties." These cells have well defined receptive fields, typically respond to only one stimulus quality or modality (i.e., hair movement), and exhibit response characteristics that remain unchanged $(10,16)$. In addition, Poggio and Mountcastle (17) showed that such neurons possess certain static properties that are unchanged by general anesthesia.

Several studies have demonstrated that some neurons in the primary somatosensory pathway are functionally mod- ulated during behavioral state. Steriade and Deschenes (19) described the development of rhythmic bursting in resting spontaneous discharges and tonically increased firing of VP neurons during brain-activated behavioral states. Other studies (15) described significant enhancement of both spontaneous and evoked multiple unit activity in specific and nonspecific thalanic nuclei when cats increased their state of arousal. Single unit recordings from the ventral posterior lateral nucleus of awake cats (1) and ventral posterior medial nucleus of awake monkeys (6) also showed changes in the spontaneous discharge of these neurons associated with shifts between waking and sleep. Single unit techniques, however, have been less successful in demonstrating arousal relationships in somatically evoked activity in VP. Hayward (6) and Baker (1) were unable to show any apparent arousalrelated changes in the discharges evoked by somatic stimuli, and only qualitative descriptions of unit responses to somatic stimuli were attempted in these studies.

The present study was designed to explore the effects of changes in the level of arousal on the unitary discharges of neurons in the ventral posterior thalamus of unanesthetized, partially restrained monkeys. The recent development, in our laboratory. of an array of calibrated. mechanical somatic

'Requests for reprints should be addressed to Thomas J. Morrow, Ph.D., V.A. Medical Center, Research 151. 2215 Fuller Rd., Ann Arbor, MI 48105. 
stimulation devices, specialized computer data acquisition hardware and software, and a real-time EEG synchronization detector has made it possible to detect and quantify subtle changes in the excitability of single somatosensory neurons during changes in behavioral state.

\section{METHOD}

\section{Surgery}

Five adult male squirrel monkeys were initially tranquilized with ketamine $\mathrm{HCl}(10 \mathrm{mg} / \mathrm{kg}$, IM) and intubated with a pediatric endotracheal tube. Animals were then deeply anesthetized using halothane in combination with nitrous oxide $(60 \%)$ and oxygen $(40 \%)$. The level of halothane was adjusted $(0.5$ to $1.0 \%)$ as needed during surgery to suppress all somatic reflexes. Under aseptic conditions, subjects were prepared for unit recording by making a $10 \mathrm{~mm}$ diameter craniotomy above the stereotaxic coordinates calculated for the exploration of the ventral posterior (VP) thalamus. A microdrive chamber (12) was positioned above the craniotomy and a stainless steel reference electrode inserted at the lateral edge of the skull opening, 2 to $3 \mathrm{~mm}$ into the underlying parietal cortex. Bipolar stainless steel stimulating electrodes, with a $2 \mathrm{~mm}$ tip separation, were implanted bilaterally under stereotaxic control into each spinal lemniscus (SL), which is comprised of the combined trajectories of the medial lemniscal and spinothalamic pathways at the midbrain level. All electrode leads were routed to an electrical connector situated on the skull, posterior to the microdrive. All electrodes, the microdrive assembly and connector were fixed to the skull using four stainless steel skull screws and dental acrylic cement. Two of these skull screws served as EEG electrodes and another as system ground. Postoperative care included administration of subcutaneous fluids to maintain hydration and routine use of systemic antibiotics (penicillin, IM, 45,000 units $/ \mathrm{kg} /$ day for 10 days).

\section{Recording and Arousal State Determination}

Subjects were allowed to recover for at least two weeks before unit recording was attempted. Each monkey was adapted to tolerate up to three hours of partial restraint in a primate chair. Based on previous work $(4,6)$, the level of arousal was divided into three categories: 1) Waking with movement (WM), where the animal is moving and low voltage fast activity dominated the EEG; 2) Quiet waking (QW), where the monkey is not moving and the EEG is desynchronized as in WM; and 3) Drowsy (D), where the EEG shows primarily high voltage, slow activity and the animal is not moving. Two parameters were continually monitored on a chart recorder and by computer to define each arousal state during single unit recording. The amplified output of a vibration sensor (weighted phonograph cartridge) attached to a flexible wand on the back of the primate chair reliably triggered output from a voltage level detector whenever the monkey produced visible movements of a body part the size of a hand or foot. Similarly, a laboratory constructed EEG synchronization detector (13) was used to register the presence of high voltage $(>50 \mu \mathrm{V})$, synchronized slow EEG activity, occurring within a frequency range of $6-17 \mathrm{~Hz}$. This device indicated the presence of synchronized EEG activity. The outputs of both EEG and movement detection circuits were monitored on separate channels of a chart recorder and led into a laboratory minicomputer along with standard pulses triggered by the discharge of single neurons (see below).

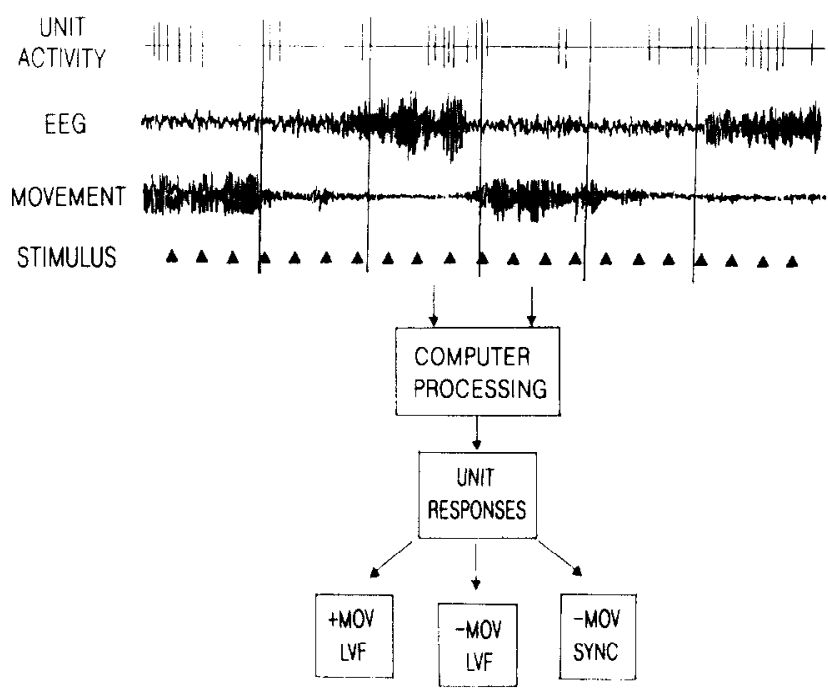

FIG. 1. Schematic representation of data acquisition during a typical unit recording session. Note how the behavioral state, defined by the EEG and movement traces, rapidly and continually changes during the period of data acquisition as a stimulus is presented. Shown in the lower boxes, computer processing (PETH software) sorts the evoked unit discharges into separate groups, based on the specific behavioral state during which the stimulus was given. The vertical lines (upper four traces) represent the divisions imposed by the software for sorting each stimulus evoked unit discharge into three distinct groups as defined by the arousal states described in the Method section. ( $\mathrm{LVF}=\mathrm{EEG}$ desynchronized with low voltage fast activity, SYNC=EEG synchronized with high voltage, slow wave activity, $\mathrm{MOV}=$ movement).

During experimental sessions, extracellular single unit activity was recorded while slowly advancing an Epoxylite ${ }^{\mathrm{TM}}$ insulated stainless steel microelectrode (5-10 Megohms impedance at $100 \mathrm{~Hz}$ ) into the somatosensory thalamus through a silicone rubber seal located at the hottom of the microdrive. The experimenter tested each unit for responses to somatic or SL stimulation as the electrode was advanced. Only well isolated action potentials (average amplitude: $586 \mu \mathrm{V} \pm 287 \mu \mathrm{V}$ SD; range: $200-2000 \mu \mathrm{V}$; signal/noise ratio $>=2 / 1$ ) were used to trigger pulses from a window discriminator (Frederich Haer Co.) with an output to a laboratory minicomputer (Data General S-140) for construction of peristimulus time histograms (PSTH). These same trigger pulses were fed into: 1) a frequency to voltage converter with analog output to the chart recorder, 2) a second channel of the oscilloscope, 3) an audio monitor, and 4) a digital counter. These monitoring devices were all used in combination to maintain the accuracy and reliability of action potential discrimination throughout each recording session.

SL stimuli were bipolar, square wave pulses (100-1000 $\mu \mathrm{A} ; 0.1 \mathrm{msec}$ duration) delivered through the midbrain electrodes at 1 to $2 \mathrm{~Hz}$. For unit testing, stimulus intensities were $2 \times$ threshold, typically placing them in $100-300 \mu \mathrm{A}$ range. We have found no evidence that low frequency SL stimulation, even at intensities of $1 \mathrm{~mA}$, had any effect on spontaneous behaviors or the pattern of EEG activity.

Somatic stimuli consisted of controlled air puffs $(0.2 \mathrm{msec}$ duration), for the selective hair stimulation, calibrated von Frey hairs (14 to $58 \mathrm{~g}$ ) for skin stimulation, manual muscle 
TABLE 1

DISTRIBUTION ARM RESPONSE CHANGES AND RESPONSE PROPERTIES OF VP NEURONS

\begin{tabular}{lccr}
\hline \multicolumn{4}{c}{ Somatically Driven Units $(\mathrm{n}=154)$} \\
\hline \# Tested for Arm & 93 & \# With Arm & 42 \\
\# Tested for & 129 & \# Responsive & 54 \\
$\begin{array}{l}\text { SL Response } \\
\text { \# SL Tested for ARM }\end{array}$ & 35 & \# SL With ARM & 15 \\
Stimulus & & \# Without ARM \\
Cutaneous & \# With ARM & & 36 \\
Deep & 26 & & 15 \\
& 16 &
\end{tabular}

$\mathrm{ARM}=$ Arousal Related Modulation, $\mathrm{SL}=$ Spinal Lemniscus.

palpation and joint manipulation. Tactile stimulation over wide areas of the body surface and SL stimulation at $1-2 \mathrm{~Hz}$ were used to search for inactive neurons. During quantitative testing, somatic stimuli were always delivered in the center of the identified receptive field.

\section{Data Analysis}

After a unit was isolated, a detailed cvaluation of the receptive field and stimulus-response characteristics were undertaken for each cell. Somatic receptive fields were mapped and adequate stimulus determined. In addition, the rate and pattern of any spontaneous discharge was noted. Quantitative testing was then begun to determine whether the somatic or SL responsiveness of the neuron was altered during shifts in the state of arousal. All data were acquired and stored by computer using a specially developed soft ware program, PETH. The use of this software was critical to the success of this project, since an animal's level of arousal changes frequently during the course of testing a single neuronal response to a given stimulus. The algorithm employed, acquired all data in such a way as to not only maintain the real-time relationship of the unit's discharge with other relevant events, but also detect spontaneous changes in the state of arousal. The PETH system is schematically illustrated in Fig. 1. The time of occurrence of all critical events, including each unit discharge, SL or somatic stimulus, and the presence of movement and EEG synchronization are computed and stored. The data are then dynamically sorted and grouped off-line so that neural responses to all stimuli presented during a specific arousal state can be separately analyzed and unit excitability determined. In addition to compiling peristimulus time histograms, this program computes the mean pre- and poststimulus spikes/stimulus, spike frequencies, pre-, postdifferences and their associated standard deviations. These data provide measures of response change and variability, so that statistical comparisons can be made between all stimulus and arousal conditions.

Histology

At the end of the final recording session, the subjects were deeply anesthetized with pentobarbital sodium (50 $\mathrm{mg} / \mathrm{kg}$ ) and transcardially perfused with saline, followed by a solution of $10 \%$ formalin. Serial 50-100 micra coronal sections through the diencephalon and midbrain were cut in the

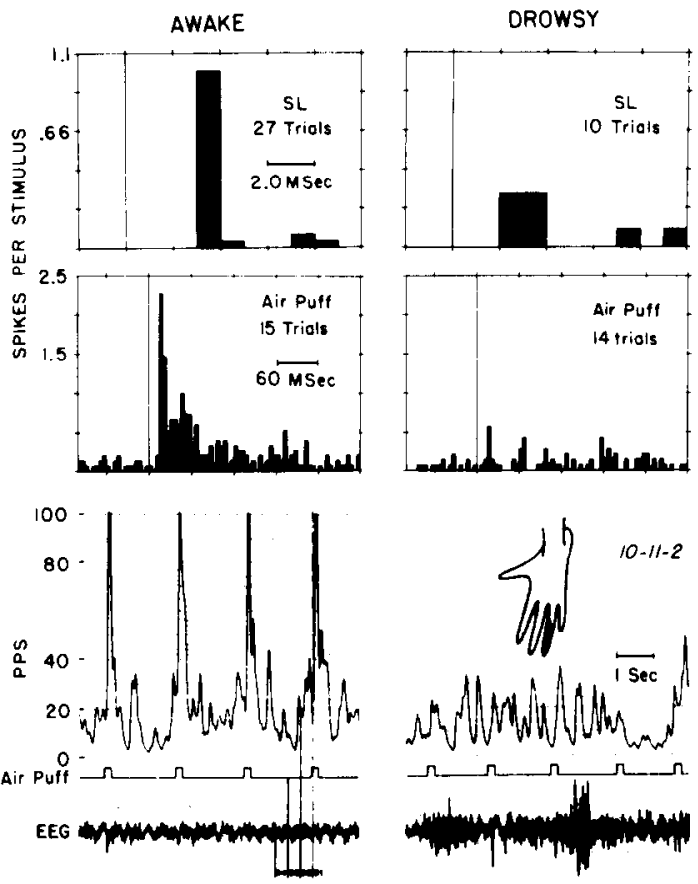

FIG. 2. VP unit responding to hair movement produced by calibrated puff of air to the contralateral receptive field on the dorsal surface of the toe (inset). Reduced responses are seen to SL stimulation (upper PSTH panels) and to somatic input (lower PSTH panels and rate meter records) during drowsy state (RIGHT) as compared to quiet waking (LEFT). Vertical line extending from top to bottom of horizontal axes in each PSTH represents time of each stimulus onset.

anterior-posterior and vertical stereotaxic planes and stained with cresyl violet. Electrode tracks and stimulation sites were reconstructed from projected images of the stained sections. Identification of unit recording sites were based on electrolytic lesions made at two or more points along each track by passing $30 \mu \mathrm{A}$ anodal DC current $(20 \mathrm{sec})$ through the microelectrode.

\section{RESULTS}

One hundred and fifty-four somatically responsive units were recorded in the lateral thalamus, including the ventral posterior lateral (VPL), medial (VPM), inferior (VPI), ventral lateral (VL) and thalamic reticular (RT) nuclei. Neurons in VL $(\mathrm{N}=9)$ and $\mathrm{RT}(\mathrm{N}=6)$ were included in our sample because they responded to somatic stimuli, were located immediately adjacent to VPL and could be studied for changes during different states of arousal. Of the total, 93 could be tested for changes in responsiveness as the monkey's level of arousal shifted among the waking with movement, quiet waking and drowsy states. Fifty-four somatically activated cclls were also reliably driven by SL stimulation, 35 of which were also tested for arousal related modulation.

We found that approximately $45 \%$ of all somatically driven VP neurons tested (42/93) exhibited changes in their somatosensory responsiveness which correlated with shifts in the state of arousal. Similarly, 15 of the $35(43 \%)$ neurons tested showed arousal related modulation of their responses to SL stimulation. Consistent with earlier reports, we also found that the spontaneous aclivity of many VP neurons 


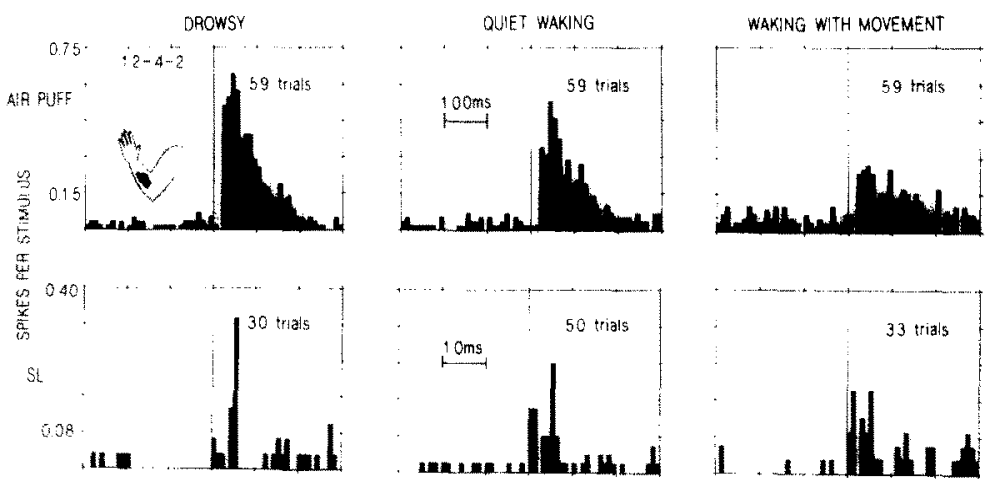

FIG. 3. VP unit responding to hair movement within the receptive field on contralateral dorsal forearm shown (inset). Both somatic (air puff stimulation, upper panels) and SL (lower panels) responses show reduced responses as the level of arousal increases from drowsy to quiet waking to waking with movement. Vertical line extending from top to bottom of horizontal axes in each PSTH represents time of stimulus onset.

varied with the level of arousal. Table 1 summarizes the response properties of the single units recorded in this study.

The majority of units exhibiting arousal related modulation of evoked activity fell primarily into two groups. The first and most predominant group $(\mathrm{N}=36)$ consisted of units that fired maximally to somatic stimuli presented during the quiet waking $(\mathrm{QW})$ state. These neurons showed decreased responsiveness during periods of either decreased (D) or increased (WM) arousal. The second and less prevalent group $(\mathrm{N}=5)$ was composed of neurons that responded best during the drowsy state, and exhibited progressively lower responses as the level of arousal increased from $D$ to $Q W$ to WM. One neuron, not included in either group above, discharged maximally during WM and with decreased responsiveness during the $\mathrm{QW}$ and $\mathrm{D}$ states.

Figure 2 illustrates the response of a typical neuron from the largest group above. Here we see the discharges of a cell to repetitive hair movements produced by an air puff stimulus applied to a contralateral receptive field on the hand. As shown in both PSTH records and chart recordings, this neuron responds maximally to stimuli presented during the awake state $(Q W)$, when the EEG was desynchronized and there was no obvious movements. When the EEG became more synchronized as the monkey became drowsy, somatic responsiveness was markedly reduced or even eliminated. It is important to note that background activity, seen in the prestimulus period, was unchanged during the time of reduced somatic responsiveness. This finding that the spontaneous discharge did not change or when changed, did not follow the direction of change(s) seen in the evoked responses was typical for many arousal modulated somatosensory neurons.

The effect of arousal state on the short latency responses elicited by SL stimulation of this same somatically activated neuron is also shown in Fig. 2. During repetitive stimulation of SL $(1 \mathrm{~Hz})$ while the monkey was in the quiet waking state, most unit discharges occurred within a narrow 1 msec wide poststimulus latency band, 3-4 $\mathrm{msec}$ after the stimulus. During drowsiness, the overall responses to the same SL stimulus were reduced and the discharges became more dispersed, occurring over the broader $2-4 \mathrm{msec}$ poststimulus period. Although not shown in Fig. 2, as the responses to SL stimulation decreased with lower levels of arousal, the spontaneous interstimulus activity of the cell was unchanged during shifts in arousal.

In contrast to the neuronal responses just described, a second group of cells exhibited evoked responses which were facilitated during drowsiness and depressed as the level of arousal increased to waking with movement. Figure 3 illustrates this type of arousal related response modulation. As in the previous example, the neuron was driven by repetitive movement of hairs within the receptive field as the contralateral limb. The histograms presented here show a depression of both the somatic and SL responses on the monkey's behavioral state changed from drowsy to quiet waking to waking with movement. Again, it is important to note that the interstimulus or prestimulus background did not follow the same trend as did the evoked response. For this unit, the spontaneous discharge in fact increased slightly with increasing arousal while at the same time the somatic and SL responses were diminished.

It was not possible to test for arousal related changes in response to both somatic and SL stimulation for all units. However, in the 35 cases where this was possible, $34 \%$ of the units (12/35) showed similar arousal related effects for both stimulus types whenever response modulation could be demonstrated for either stimulus alone. Only 3 neurons exhibited modulation of the response to peripheral somatic stimulation exlusively. No units showed arousal related modulation of the SL responses alone.

Only one neuron could be adequately tested for changes in the size of its receptive field (RF) as a function of the state of arousal. Arousal related modulation of both the relative magnitude of its discharge to a somatic stimulus and the size of its receptive field was found for this neuron. The cell had a large receptive field that included a $14 \times 0.6 \mathrm{~cm}\left(8.4 \mathrm{~cm}^{2}\right)$ area on the tail during quiet waking (QW), and showed a reduction in field area to approximately $0.3 \mathrm{~cm}^{2}$ when the monkey became drowsy (D).

The response properties of all somatic units tested are summarized in Table 1. The data presented here show that the likelihood of occurrence of arousal-related response 
modulation in a given VP somatosensory neuron cannot be predicted on the basis of whether it receives input from the cutaneous or deep structures $\left(\chi^{2}=0.4396\right)$. No differences in ARM were found across all response properties, regardless of the particular adequate stimulus (hair. cutaneous, muscle, etc.).

\section{DISCUSSION}

Early studies examining the physiology of VP thalamus described the majority of VP neurons as having discharge properties that remained static and unchanged with changes in behavioral state, including general anesthesia (16). However, we have shown that a large proportion of somatically driven ventral posterior thalamic neurons possess discharge characteristics that would classify them as markedly dynamic and varying with behavioral state. These VP neurons showed changes in their spontaneous and evoked responses that are correlated with changes in the level of arousal as defined by objective electroencephalographic and behavioral criteria. Of those cells that showed arousal related modulation, most responded maximally to a somatic or SL stimulus when the monkey was in the quiet waking state. A smaller population of neurons showed a complementary type of arousal related modulation in that they responded best when the state of arousal was at its lowest and decreased responsiveness as arousal increased.

These data are in contrast with the findings of Baker (1) and Hayward (6) who were unable to demonstrate any effect of arousal on the evoked discharges of single neurons in the VP of awake cat and monkey, respectively. It is not clear why such lindings were missed in these studies, because the response modulation which we saw in many units was sufficiently robust to be seen even in the chart record output of our ratemeter. One possible reason for this discrepancy might be interstimulus variability of somatic stimuli when testing unit responses during the different arousal states.

It is also possible that differences seen in these studies could relate to the specific behavioral states present during which testing was performed. The investigations of both Baker and Hayward focused on the changes occurring during sleep and waking, rather than on the stages of arousal preceding sleep. It is possible that there can be no direct comparison of their findings and those of this study, because our Inonkeys did not sleep. It may be that neuronal sensory responsiveness during sleep is more like that during quiet waking than in the drowsy state. Such a hypothesis in fact gains support from the work of Favale et al. (5), who showed that evoked potentials recorded in the cat fluctuated during the sleeping-waking cycle with greater amplitude during sleep than during waking.

Our data provide evidence that the size of the somatic receptive field of a neuron can also vary as a function of arousal. While only one such neuron was found in this study, this may be due to the technical difficulty involved in accurately measuring changes in small receptive fields in the awake animal. Only cells with large receptive fields could be adequately tested for such changes. It is possible that many of the units showing ARM in the relative magnitude of their somatically evoked discharge also had changes in receptive field size, which were undetected. Slight changes in response latency might also be missed.

The data presented here suggest that at least one site for the modulation of VP responses is at this thalamic level. SL stimulation bypasses spinal and dorsal column inputs and evokes short latency responses in VP neurons that involve only one or two synaptic relays. The activation of descending modulatory or collateral pathways does not influence our results because the short response latencies do not allow enough time for such long conduction delays. Arousal related modulation for $\mathrm{SL}$ responses must therefore occur within or very near the ventral posterior thalamus. Additional sites for arousal related modulation of VP thalamus cannot be ruled out. While most VP neurons showed ARM for responses elicited by both peripheral and SL stimulation, three units failed to exhibit modulation of both stimuli and showed exlusive modulation of the responses to somatic stimuli. This suggests other possible pathways or mechanisms in addition to direct thalamic action on VP neurons.

Other evidence suggests that arousal related response modulation is input specific and not the result of an overall change in the postsynaptic excitability of a VP thalamic neuron. If changes had occurred in the overall excitability of a neuron, then similar changes would be expected in both the evoked and spontaneous activity of the cell. Most VP neurons, however, showed no change or an opposite change in spontaneous activity relative to those changes obtained during the ARM of somatic and SL elicited discharges. These data therefore suggest an input specific modulation at the thalamic level, possibly by action on the distal portion of the dendrites of VP neurons. The role of local circuit interneurons within VP can also not be ruled out. Presynaptic inhihitory mechanisms involving axo-axonal contacts are unlikely because the anatomical basis for such inhibition is lacking in the primate thalamus (14).

The sensory responses of thalamic neurons could also be directly influenced by the activity of cortical, diencephalic or brainstem extralemniscal neurons that project to the somatosensory thalamic nuclei. The anatomical basis for direct corticothalamic control has been demonstrated $(8,9)$. Reccntly, we described corticofugal influences on the responses of ventrobasal (VB) thalamic neurons in anesthetized and awake rat $(21,22)$. We demonstrated that focal suppression of $S 1$ cortex reduced the number of discharges of VB neurons in response to electrical stimulation of the medial lemniscus or the somatic receptive field. These data provide evidence that somatosensory transmission to VB neurons is primarily facilitated by $\mathrm{S} 1$ corticothalamic neurons.

Bowsher (2) also reviewed evidence for reticular formation and tectal inputs to somatosensory thalamus. Scheibel et al. (18) and others suggest an anatomical substrate for modulation of the thalamic ventrobasal complex via connections with both cortex and the adjacent thalamic reticular nucleus. Anatomical studies show a projection of cortical efferents to VB and to the thalamic reticular nucleus that parallels the topographic projection to the thalamic relay nuclei $(7,11,19)$. Thus the thalamic reticular nucleus receives inputs from the cerebral cortex organized so that any cortical area projects to the thalamic reticular and neighboring VB complex with these two corticothalamic sites interconnected by thalamo-reticular and reticulo-thalamic fibers $(3,9,11)$.

In summary, there is considerable evidence that many systems may be involved in the modulation of somatosensory transmission through the VP thalamus. The underlying mechanisms responsible for changes in VP responsiveness during shifts in arousal as reported here are yet to be determined. 


\section{ACKNOWLEDGEMENTS}

We would like to thank Patricia M. Morris for her superior technical assistance with data acquisition, analysis and figure preparation for this work. Funded in part by grants from the Air Force and NIH.

\section{REFERENCES}

1. Baker, M. A. Spontaneous and evoked activity of neurons in the somatosensory thalamus of the waking cat. J. Physiol. 217:359-379; 1971.

2. Bowsher, D. Diencephalic projections from the midbrain reticular formation. Brain Res. 95:211-220; 1975.

3. Carman, J. B.; Cowan, W. M.; Powell, T. S. Cortical connections of the thalamic reticular nucleus. J. Anat. 98:588-598; 1964.

4. Casey, K. L. Unit analysis of nociceptive mechanisms in the thalamus of the awake squirrel monkey. J. Neurophysiol. 29:727-750; 1966 .

5. Favale, E.; Loeb, C.; Manfredi, M.; Giandomenico, S. Somatic afferent transmission and cortical responsiveness during natural sleep and arousal in the cat. Electroencephalogr. Clin. Neurophysiol. 18:354-368; 1965.

6. Hayward, J. M. Response of ventrobasal thalamic cells to hair displacement on the face of the waking monkey. J. Physiol. 250:385-407; 1975.

7. Jones, E. G. Some aspects of the organization of the thalamic reticular complex. J. Comp. Neurol. 162:285-308; 1975.

8. Jones, E. G.; Powell, T. P. S. Connections of the somatic sensory cortex of the Rhesus monkey. III. Thalamic connections. Brain 93:37-56; 1970.

9. Jones, E. G.; Wise, S. P.; Coulter, J. D. Differential thalamic relationships of sensory-motor and parietal cortical fields in monkeys. J. Comp. Neurol. 183:833-882; 1979.

10. Loe, P. R.; Whitsel, B. L.; Dreyer, D. A.; Metz, C. B. Body representation in ventrobasal thalamus of Macaque: A singleunit analysis. J. Neurophysiol. 40:1339-1355; 1977.

11. Minderhoud, J. M. An anatomical study of the efferent connections of the thalamic reticular nucleus. Exp. Brain Res. 12:435$446 ; 1971$.
12. Morrow, T. J. An improved technique for recording single unit activity in awake animals. Brain Res. Bull. 5:91-93; 1980.

13. Morrow, T. J.; Casey, K. L. An automated device for the realtime detection of alpha and spindle activity in the EEG. Brain Res. Bull. 16:439-442: 1986.

14. Ohara, P. T.; Ralston, H. J., III; Ralston, D. D. The morphology of neurons and synapses in the somatosensory thalamus of the cat and monkey. In: The thalamus and pain. Amsterdam, Netherlands: Elsevier Science Publ. B.V.; 1987:171-184.

15. Podvoll, E. M.; Goodman, S. J. Averaged neural electrical activity and arousal. Science 155:223-225; 1967.

16. Poggio, G. F.; Mountcastle, V. B. A study of the functional contributions of the lemniscal and spinothalamic systems to somatic sensibility. Bull. Johns Hopkins Hosp. 108:266-316; 1960.

17. Poggio, G. F.; Mountcastle, V. B. The functional properties of ventrobasal thalamic neurons studied in unanesthetized monkeys. J. Neurophysiol. 26:775-806; 1963.

18. Scheibel, M. E.; Scheibel, A. B.; Davis, T. H. Some substrates for corticofugal control over thalamic cell ensembles. In: Corticuthalamic projections of sensorimotor activies. New York: Raven Press; 1972:131-160.

19. Scheibel, M. E.; Scheibel, A. B. Organization of the nucleus reticularis thalami: A golgi study. Brain Res. 1:43-62;1966.

20. Steriade, M.; Deschenes, $M$. The thalamus as a neural oscillator. Brain Res. Rev. 8:1-63; 1984.

21. Yuan, B.; Morrow, T. J.; Casey, K. L. Responsiveness of ventrobasal thalamic neurons after focal suppression of SI cortex in the anesthetized rat. J. Neurosci. 5:2971-2978: 1985.

22. Yuan, B.; Morrow, T. J.; Casey, K. L. Corticofugal influences of SI cortex on ventrobasal thalamic neurons in the awake rat. J. Neurosci. 6:3611-3617: 1986. 